65

\title{
Collaborative work using our Virtual Reality Network System (VLNET)
}

\author{
Nadia Magnenat Thalmann, Igor Pandzic \\ MIRALab - CUI, University of Geneva \\ 24 rue du Général-Dufour \\ CH1211 Geneva 4, Switzerland \\ tel. +41-22-705 $7769 ;$ fax +41-22-705 7780 \\ \{Nadia.Thalmann,Igor.Pandzic\}@cui.unige.ch \\ http://miralabwww.unige.ch/
}

\begin{abstract}
Networked Collaborative Virtual Environments (NCVE) are systems that allow several geographically distant users to collaborate within the common 3D virtual environment. The users become part of the environment represented by graphical embodiments and they can interact with the environment and with each other. We analyze the importance of good graphical representation of each user for communication and collaboration. The Virtual Life Network (VLNET) system is an NCVE using sophisticated Virtual Humans as user embodiments to improve collaboration. We describe the system and some experimental applications.
\end{abstract}

Keywords

Networked Collaborative Virtual Environments, Collaboration, Virtual Humans

\section{INTRODUCTION}

Networked Collaborative Virtual Environments (NCVEs) are systems that allow multiple geographically distant users to evolve in a common virtual environment, interact with it and with each other. Each workstation has a copy of the virtual environment. All events that have an impact on the environment are transmitted to other sites so that all environments can be updated and kept consistent, giving the impression for the users of being in the same, unique environment. The users 
become a part of the environment, embodied by a graphical representation that should ideally be human-like.

Networked Collaborative Virtual Environment (NCVE) systems are suitable for numerous collaborative applications ranging from games to medicine (Doenges, 1997), for example:

- Virtual teleconferencing with multimedia object exchange

- All sorts of collaborative work involving 3D design

- Multi-user game environments

- Teleshopping involving 3D models, images, sound (e.g. real estate, furniture, cars)

- Medical applications (distance diagnostics, virtual surgery for training)

- Distance learning/training

- Virtual Studio/Set with Networked Media Integration

- Virtual travel agency.

The NCVE systems are often described as systems that permit to the users to feel as if they were together in a shared Virtual Environment. Indeed, the feeling of "being together" is extremely important for collaboration, as well as for the sense of presence felt by the subjects. A very important factor for the feeling of being together in a virtual world is the way users perceive each other: their embodiment. In a broader sense, this includes not just the graphical appearance but also the way movements, actions and emotions are represented.

In the following section we discuss the importance of the user representation in NCVEs. After that we describe the Virtual Life Network (VLNET), a NCVE system using Virtual Humans for user representation. In the results section we describe several experimental applications of our system.

\section{THE IMPORTANCE OF VIRTUAL HUMANS IN NCVE}

The participant representation in a networked VE system has several functions:

- perception (to see if anyone is around)

- localization (to see where the person is)

- identification (to recognize the person)

- visualization of interest focus (to see where the person's attention is directed)

- visualization of actions (to see what the person is doing)

- communication (lip movement in synchronization with speech, facial expressions, gestures).

Virtual Humans can fulfill all these functions in an intuitive, natural way resembling the way we achieve these tasks in real life. Even with limited sensor information, a virtual human frame can be constructed in the virtual world, reflecting the activities of the real user. Slater and Usoh (Slater, 1994) indicate that such a body, even if crude, already increases the sense of presence that the 
participants feel. The participants visualize the environment through the eyes of their virtual actor, and move their virtual body by different means of body control. In addition, introducing the human-like autonomous actors for various tasks increases the level of interaction within the virtual environment.

\section{VIRTUAL LIFE NETWORK}

Based on the considerations from the previous section we have developed the Virtual Life Network (VLNET) system (Capin 1997). From the networking point of view, VLNET is based on a fairly simple client/server architecture. Next subsections discuss in more detail the server and the client architecture.



Figure 1 Virtual Life Network system overview. 


\subsection{VLNET Server}

A VLNET server site consists of a HTTP server and a VLNET Connection Server. They can serve several worlds, which can be either VLNET files or VRML 1.0 files. For each world, a World Server is spawned as necessary, i.e. when a client requests a connection to that particular world. The life of a World Server ends when all clients are disconnected.

A VLNET session is initiated by a Client connecting to a particular world designated by a URL. The Client first fetches the world database from the HTTP server using the URL. After that it extracts the host name from the URL and connects to the VLNET Connection Server on the same host. The Connection Server spawns the World Server for the requested world if one is not already running and sends to the Client the port address of the World Server. Once the connection is established, all communication between the clients in a particular world passes through the World Server.

\subsection{VLNET Client}

The design of the VLNET Client is highly modular, with functionalities split into a number of processes. Figure 1 presents an overview of the modules and their connections. VLNET has an open architecture, with a set of interfaces allowing a user with some programming knowledge to access the system core and change or extend the system by plugging custom-made modules, called drivers, into the VLNET interfaces. In the next subsections we explain in some detail the VLNET Core with its various processes, as well as the drivers and the possibilities for system extension they offer.

\subsubsection{VLNET Core}

The VLNET core is a set of processes, interconnected through shared memory that perform basic VLNET functions. The Main Process performs higher level tasks, like object manipulation, navigation, body representation, while the other processes provide services for networking (Communication Process), database loading and maintenance (Database Process) and rendering (Cull Process and Draw Process).

The Main Process consists of five logical entities, called engines, covering different aspects of VLNET. It also initializes the session and spawns all other processes and drivers. Each engine is equipped with an interface for the connection of external drivers.

The Object Behavior Engine takes care of the predefined object behaviors, like rotation or falling. The Navigation and Object Manipulation Engine takes care of the basic user input: navigation, picking and displacement of objects. The Body Representation Engine is responsible for the deformation of the body. In any given body posture (defined by a set of joint angles) this engine will provide a deformed body ready to be rendered. The body representation is based on the Humanoid body model (Boulic, 1995). This engine provides the interface for changing the 
body posture. A standard Body Posture Driver is provided, that connects also to the navigation interface to get the navigation information, then uses the Walking Motor and the Arm Motor (Boulic, 1990, Pandzic, 1996) to generate the natural body movement based on the navigation. The Facial Representation Engine provides the synthetic faces with a possibility to change expressions or the facial texture. The facial representation is a polygon mesh model with Free Form Deformations simulating muscle actions (Kalra, 1992). The Video Engine manages the streaming of dynamic textures to the objects in the environment and their correct mapping.

All the engines in the VLNET core process are coupled to the main shared memory and to the message queue.

Cull and Draw Processes access the main shared memory and perform the functions of culling and drawing as their names suggest. These processes are standard SGI Performer (Rohlf, 1994) processes.

The Communication Process handles all network connections and communication in VLNET. All other processes and engines are connected with it through the Message Queue. They fill the queue with outgoing messages for the Communication Process to send. The messages are sent to the server, distributed and received by Communication Processes of other clients. The Communication Process puts these incoming messages into the Message Queue from where the other processes and engines can read them and react.

All messages in VLNET use the standard message packet. The packet has a standard header determining the sender and the message type, and the message body. The message body content depends on the message type but is always of the same size ( 80 bytes), satisfying all message types in VLNET. The video data from the Video Engine is a special case and is handled using a separate communication channel. It is given lower priority than the other data. By isolating the communications in this separate process, and by keeping the VLNET Server relatively simple, we leave the possibility to switch relatively easily to a completely different network topology, e.g. multicasting instead of client/server.

The Data Base Process takes care of the off-line fetching and loading of objects and user representations. By keeping these time consuming operations in the separate process non-blocking operation of the system is assured.

\subsubsection{VLNET Drivers}

The drivers provide the simple and flexible means to access and control all the complex functionalities of VLNET. Simple, because each driver is programmed using a very small API that basically consists of exchanging crucial data with VLNET through shared memory. Flexible, because using various combinations of drivers it is possible to support all sorts of input devices ranging from the mouse to the camera with complex gesture recognition software, to control all the movements of the body and face using those devices, to control objects in the environment and stream video textures to them, to build any amount of artificial intelligence in order to produce autonomous or semi-autonomous virtual humans in the networked virtual environment. 
The Drivers are directly tied to the Engines in the VLNET Main Process. Each engine provides a shared memory interface to which a driver can connect. Most drivers are optional and the system will provide minimal functionality (plain navigation and manipulation of objects) without any drivers.

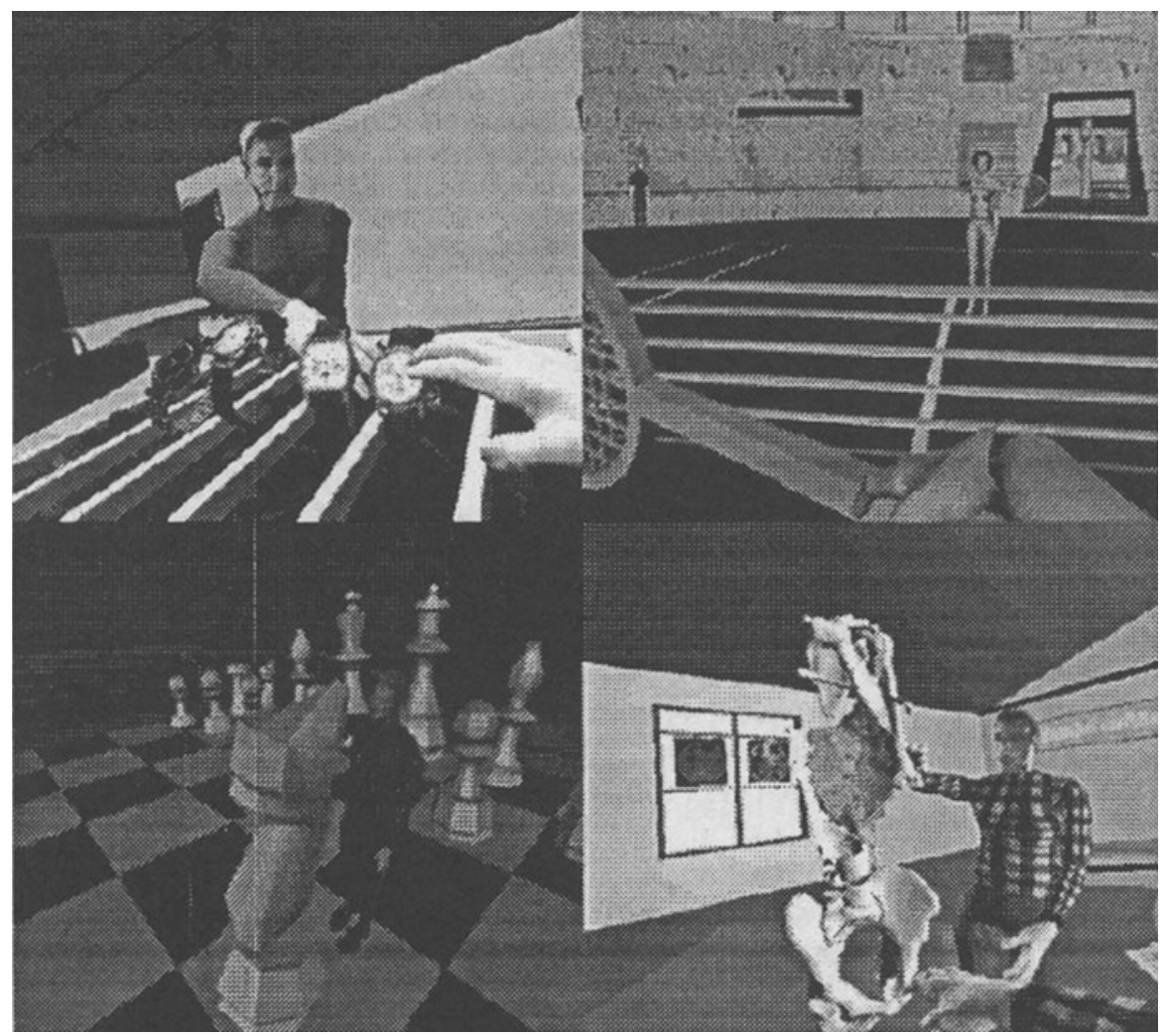

Figure 2 Snapshots from applications.

\section{RESULTS}

Several experimental applications were developed using the VLNET system. Some snapshots are presented in Figure 2.

\subsection{Entertainment}

NCVE systems lend themselves to development of all sorts of multi user games. We had successful demonstrations of chess and other games played between Switzerland and Singapore, as well as between Switzerland and several European countries.

A virtual tennis game has been developed (Noser, 1996) where the user plays against an opponent who is an autonomous virtual human. The referee is also an 
autonomous virtual human capable of refereeing the game and communicating the points, faults etc. by voice.Currently a multi user adventure game is under development.

\subsection{Teleshopping}

In collaboration with Chopard, a watch company in Geneva, a teleshopping application was successfully demonstrated between Geneva and Singapore. The users were able to communicate with each other within a rich virtual environment representing a watch gallery with several watches exposed. They could examine the watches together, exchange bracelets, and finally choose a watch.

\subsection{Medical education}

In collaboration with our colleagues working on a medical project we have used the 3D data of human organs, muscles and skeleton reconstructed from MRI images (Beylot, 1996) to build a small application in the field of medical education. The goal is to teach a student the position, properties and function of a particular muscle. In the virtual classroom several tools are at the disposition of the professor. MRI, CT and anatomical slice images are on the walls showing the slices where the muscle is visible. A $3 \mathrm{D}$ reconstruction of the skeleton is available together with the muscle, allowing to examine the shape of the muscle and the points of attachment to the skeleton. Finally, an autonomous virtual human with a simple behavior is there to demonstrate the movements resulting from a contraction of the discussed muscle.

\subsection{Stock exchange}

Currently an application is being developed to visualize in 3D the real time updates of stock exchange data and allow interactions of users with the data and with each other.

\section{CONCLUSIONS}

We have discussed problems involved with introducing high level human representations in the Networked Collaborative Virtual Environments and presented one solution to this problem - the Virtual Life Network. The system's modular architecture, the function of each component and their interconnections were described in detail. Various experimental applications highlighted in the results section show that this kind of system lends itself to a wide variety of collaborative applications. 


\section{ACKNOWLEDGMENTS}

This research is financed by "Le Programme Prioritaire en Telecommunications de Fonds National Suisse de la Recherche Scientifique" and the TEN-IBC project VISINET.

\section{REFERENCES}

Beylot P., Gingins P., Kalra P., Magnenat-Thalmann N., Maurel W., Thalmann D., Fasel J. (1996) 3D Interactive Topological Modeling using Visible Human Dataset, Proceedings of EUROGRAPHICS 96, Poitiers, France

Boulic R., Magnenat-Thalmann N. M.,Thalmann D. (1990) A Global Human Walking Model with Real Time Kinematic Personification, The Visual Computer, Vol.6(6)

Boulic R., Capin T., Huang Z., Kalra P., Lintermann B., Magnenat-Thalmann N., Moccozet L., Molet T., Pandzic I., Saar K., Schmitt A., Shen J., Thalmann D. (1995) The Humanoid Environment for Interactive Animation of Multiple Deformable Human Characters, Proceedings of Eurographics ' 95

Capin T.K., Pandzic I.S., Noser H., Magnenat Thalmann N., Thalmann D. (1997)

Virtual Human Representation and Communication in VLNET Networked

Virtual Environments, IEEE Computer Graphics and Applications, Special Issue on Multimedia Highways

Doenges P.K., Capin T.K., Lavagetto F., Ostermann J., Pandzic I.S., Petajan E.D. (1997) MPEG-4: Audio/Video \& Synthetic Graphics/Audio for Mixed Media, Image Communication Journal, Special issue on MPEG-4

Kalra P., Mangili A., Magnenat Thalmann N., Thalmann D. (1992) Simulation of Facial Muscle Actions Based on Rational Free Form Deformations, Proc. Eurographics '92, pp.59-69.

Noser H., Pandzic I.S., Capin T.K., Magnenat Thalmann N., Thalmann D. (1996) Playing Games through the Virtual Life Network, Proceedings of Artificial Life $V$, Nara, Japan

Pandzic I.S., Capin T.K., Magnenat Thalmann N., Thalmann D. (1996) Motor functions in the VLNET Body-Centered Networked Virtual Environment, Proc. of 3rd Eurographics Workshop on Virtual Environments, Monte Carlo

Rohlf J., Helman J., (1994) IRIS Performer: A High Performance Multiprocessing Toolkit for Real-Time 3D Graphics", Proc. SIGGRAPH'94

Slater M., Usoh M. (1994) Body Centered Interaction in Immersive Virtual Environments", in Artificial Life and Virtual Reality, N. Magnenat Thalmann, D. Thalmann, eds., John Wiley, pp 1-10 


\section{BIOGRAPHIES}

Nadia Magnenat Thalmann founded Miralab, a Research Lab at the University of Geneva, in 1989. She is a pioneering researcher in the field of virtual humans and has authored or co-authored more then 200 scientific papers. She has received several awards, including the 1985 Communications Award from the Government of Quebec, the 1992 Moebius Award from the European Community, and the British Computer Science Award in 1993. She received a PhD in quantum physics and computer graphics from the University of Geneva.

Igor S. Pandzic is a PhD student at Miralab, University of Geneva. His research interests include collaborative virtual environments, parallel computing, computergenerated film production and real time facial expression analysis and synthesis. He finished his undergraduate studies at the University of Zagreb in 1993. and obtained a Masters degree in computer graphics from the Swiss Federal Institute of Technology in Lausanne in 1994. 